\title{
Cytoskeletal Requirements in Axonal Transport of Slow Component-b
}

\author{
Subhojit Roy, ${ }^{1,2}$ Matthew J. Winton, ${ }^{1}$ Mark M. Black, ${ }^{4}$ John Q. Trojanowski, ${ }^{1,2,3}$ and Virginia M.-Y. Lee ${ }^{1,2,3}$ \\ ${ }^{1}$ Center for Neurodegenerative Disease Research, ${ }^{2}$ Department of Pathology and Laboratory Medicine, and ${ }^{3}$ Institute on Aging, University of Pennsylvania \\ School of Medicine, Philadelphia, Pennsylvania 19104, and ${ }^{4}$ Department of Anatomy and Cell Biology, Temple University Hospital, Philadelphia, \\ Pennsylvania 19130
}

\begin{abstract}
Slow component-b (SCb) translocates $\sim 200$ diverse proteins from the cell body to the axon and axon tip at average rates of $\sim 2-8 \mathrm{~mm} / \mathrm{d}$. Several studies suggest that SCb proteins are cotransported as one or more macromolecular complexes, but the basis for this cotransport is unknown. The identification of actin and myosin in SCb led to the proposal that actin filaments function as a scaffold for the binding of other SCb proteins and that transport of these complexes is powered by myosin: the "microfilament-complex" model. Later, several SCb proteins were also found to bind F-actin, supporting the idea, but despite this, the model has never been directly tested. Here, we test this model by disrupting the cytoskeleton in a live-cell model system wherein we directly visualize transport of SCb cargoes. We focused on three SCb proteins that we previously showed were cotransported in our system: $\alpha$-synuclein, synapsin-I, and glyceraldehyde-3phosphate dehydrogenase. Disruption of actin filaments with latrunculin had no effect on the velocity or frequency of transport of these three proteins. Furthermore, cotransport of these three SCb proteins continued in actin-depleted axons. We conclude that actin filaments do not function as a scaffold to organize and transport these and possibly other SCb proteins. In contrast, depletion of microtubules led to a dramatic inhibition of vectorial transport of SCb cargoes. These findings do not support the microfilament-complex model, but instead indicate that the transport of protein complexes in $\mathrm{SCb}$ is powered by microtubule motors.
\end{abstract}

Key words: axonal transport; slow transport; slow component-b; $\alpha$-synuclein; synapsin-I; protein complexes

\section{Introduction}

Axonal transport conveys proteins from the neuronal perikarya into axons and axon terminals in two overall rate classes, fast and slow axonal transport. The fast component (FC) delivers mainly membrane-bound organelles at average rates of $50-400 \mathrm{~mm} / \mathrm{d}$. Slow axonal transport consists of two fairly distinct subcomponents termed slow components a and b (SCa and SCb, respectively). SCa transports microtubules and neurofilaments at average rates of $\approx 1 \mathrm{~mm} / \mathrm{d}$. SCb moves at average rates of $2-8 \mathrm{~mm} / \mathrm{d}$ and transports $\approx 200$ proteins, of which only a handful have been identified. Among these are myosin, dynein, clathrin, calmodulin, synapsin, $\alpha$-synuclein, glyceraldehyde-3-phosphate dehydrogenase (GAPDH), superoxide dismutase-1, phosphofructokinase, ubiquitin, molecular chaperones, tau and other

\footnotetext{
Received Jan. 23, 2008; revised March 23, 2008; accepted April 9, 2008.

This work was supported by National Institute on Aging Grant AG09215 and by a grant from the Picower Foundation and the Benaroya family. S.R. was supported by a postdoctoral grant from the American Parkinson's Disease Association. We thank Chi Li for his technical expertise with the CDNA constructs, Dr. C. Kaether (LudwigMaximilians-University, Munich, Germany) for the APP:::YFP construct, Dr. G. Augustine (Duke University, Durham, NC) for the GFP::synapsin-I construct, and Dr. J. Bereiter-Hahn (Kinematic Cell Research Group, Goethe University, Frankfurt, Germany) for the GFP::GAPDH construct.

Correspondence should be addressed to Dr. Virginia M.-Y. Lee, Center for Neurodegenerative Disease Research, Department of Pathology and Laboratory Medicine, University of Pennsylvania School of Medicine, 3600 Spruce Street, 3rd floor, Maloney Building, Philadelphia, PA 19104. E-mail: vmylee@mail.med.upenn.edu.

S. Roy's present address: Department of Neurosciences, 9500 Gilman Drive, University of California, San Diego, San Diego, CA 92037.

D01:10.1523/JNEUROSC1.0309-08.2008

Copyright $\odot 2008$ Society for Neuroscience $\quad$ 0270-6474/08/285248-09\$15.00/0
}

microtubule-associated proteins (MAPs), spectrin, actindepolymerizing factor, and actin (Willard et al., 1974; Black and Lasek, 1979; Brady et al., 1981; Garner and Lasek, 1982; Lasek et al., 1984; Baitinger and Willard, 1987; Nixon et al., 1990; Bray et al., 1992; Mercken et al., 1995; Dillman et al., 1996; Yuan et al., 1999; Ma et al., 2000; Bourke et al., 2002; Li et al., 2004). Clearly, the proteins of $\mathrm{SCb}$ are functionally diverse, play critical roles in maintaining the integrity of axons and synapses, and are implicated in neurodegenerative diseases (Roy et al., 2005). Early radiolabeling studies suggested that many SCb proteins move together in the axons as if they are components of a common cargo (Willard et al., 1974; Black and Lasek, 1980; Tytell et al., 1981; Garner and Lasek, 1982; Yuan et al., 1999). More recently, using time-lapse imaging, we have shown that the SCb proteins $\alpha$-synuclein, synapsin-I, and GAPDH are cotransported in axons as components of a discrete complex (Roy et al., 2007). In the present study, we explore some of the mechanisms underlying $\mathrm{SCb}$ transport.

The identification of actin in SCb (Willard et al., 1974; Black and Lasek, 1979) led to the proposal that actin filaments function in SCb transport in two different contexts. First, F-actin and its motor myosin could power the transport of SCb. The identification of myosin in SCb (Willard, 1977) provided additional support for this notion. Second, F-actin could function as a carrier/ scaffold for the transport of other SCb proteins. The observations that proteins of SCb (but not SCa or FC) are concentrated in the actin-rich subaxolemmal region of the axon (Levine and Willard, 
A. Variable pauses in $\alpha$-synuclein transport

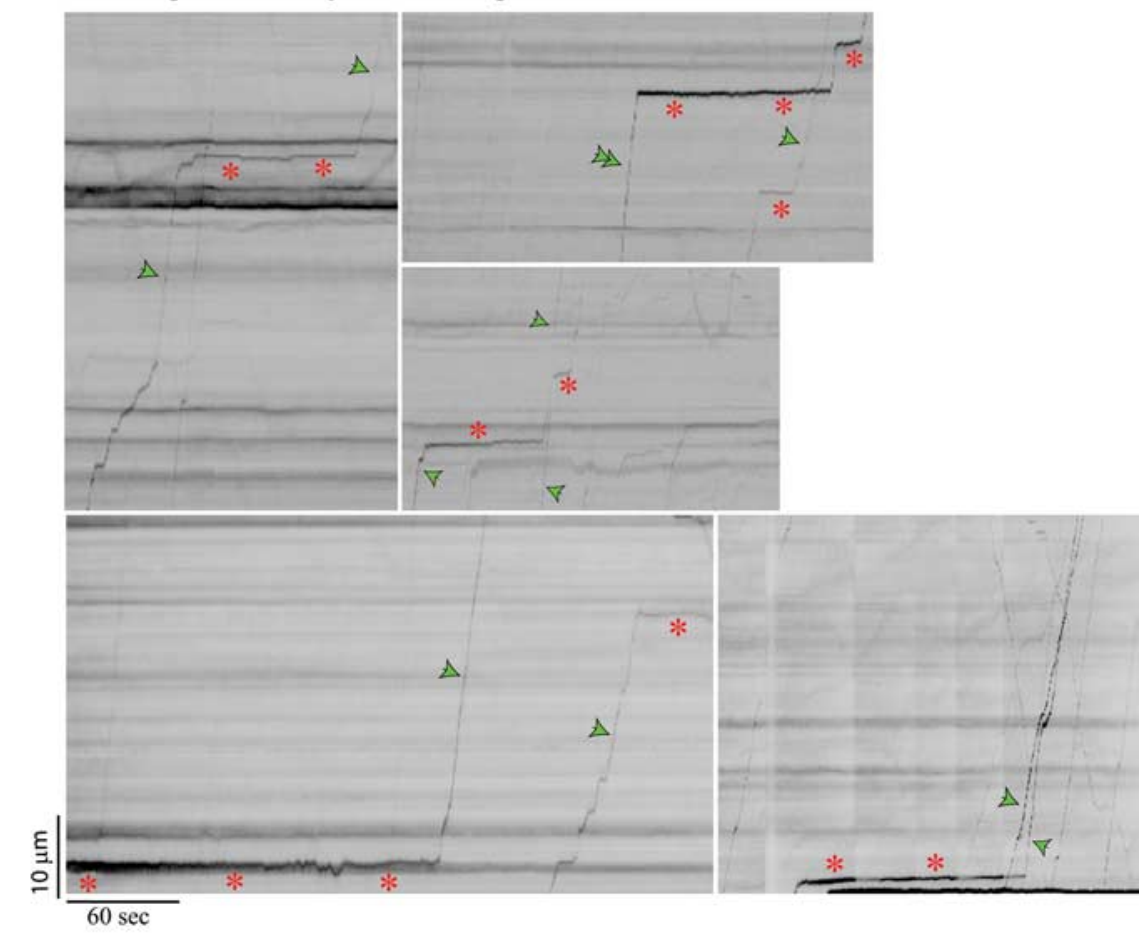

B. Differential dynamics of slow and fast transport mRFP:: $\alpha$-syn
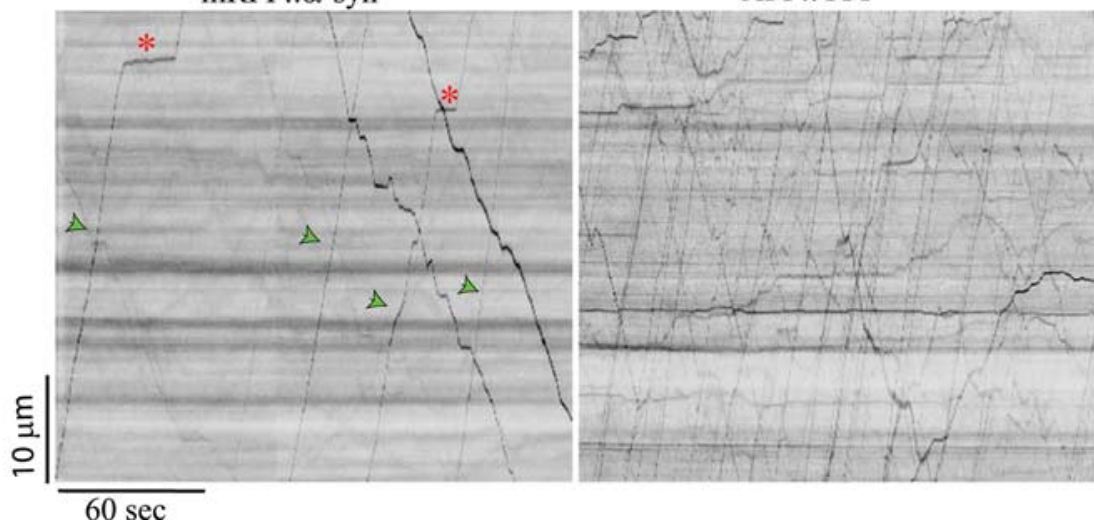

$60 \mathrm{sec}$

Figure 1. Dynamics of $\alpha$-synuclein transport in SCb. A, Cultured hippocampal neurons were transfected with mRFP:: $\alpha$-SYN, and transport was visualized in thin axons with low diffuse fluorescence. Kymographs generated from such movies are shown. Diagonal lines from bottom left to top right denote anterograde movement; pauses are denoted by horizontal lines. Anterograde $\alpha$-synuclein particles paused for varying durations (red asterisks) between spurts of movement (green arrowheads). Whereas some particles only paused transiently, others were paused for the majority of the time imaged before being transported again (bottom). A few particles showed both long and short pauses (double arrowhead). B, Comparison of kymographs of $\alpha$-synuclein transport in SCb compared with a classic fast-transport marker, APP tagged with YFP. Note the marked difference in the transport frequencies of the $\alpha$-synuclein and APP. Scale bars for kymographs are shown at the bottom left.

1981; Heriot et al., 1985) and that several SCb proteins, including synapsin, spectrin, GAPDH, clathrin, and heat shock proteins, can bind to actin filaments (Bahler and Greengard, 1987; Minaschek et al., 1992; Moss et al., 1992) further support the idea. Based on early observations, the "microfilament-complex" model was proposed, in which actin filaments serve as a scaffold to which SCb proteins bind directly or indirectly, and the complex of F-actin and associated SCb proteins is transported within the axon, powered by myosin (Garner and Lasek, 1981). Indeed, actin does perform scaffolding functions in a variety of cellular contexts, including presynaptic boutons (Sankaranarayanan et al., 2003), lending credence to the idea. However, the microfilament-complex model is based entirely on circumstantial evidence, and other indirect data have been used to argue against this model (Bray et al., 1992; Yuan et al., 1999). With the development of live-cell model systems, we can now directly test the role of F-actin in SCb transport. In this study, we determined the effects of F-actin disruption on the transport of SCb cargoes and thereby directly tested essential tenets of the microfilamentcomplex model.

\section{Materials and Methods}

Immunofluorescence and cDNA constructs. For immunofluorescence studies of mouse and human $\alpha$-synuclein, an affinity-purified rabbit polyclonal (SNL-1) was used (Giasson et al., 2000). To detect microtubules and actin filaments, we used an antibody to tubulin (DM1- $\alpha$; Sigma, St. Louis, MO) and Alexa-488 and 594labeled phalloidin (Invitrogen, Carlsbad, CA). For detecting $\alpha$-synuclein, neurons were fixed with $4 \%$ paraformaldehyde and $120 \mathrm{~mm}$ sucrose for $10 \mathrm{~min}$, extracted with $0.25 \%$ Triton X-100, blocked with $1 \%$ bovine serum albumin and $5 \%$ fetal bovine serum, and then incubated with the primary and secondary antibodies. To visualize actin and microtubules optimally, we simultaneously fixed/extracted neurons with paraformaldehyde/glutaraldehyde in a cytoskeletonstabilizing buffer as described previously (Slaughter et al., 1997). The cDNA constructs, monomeric red fluorescent protein tagged to $\alpha$-synuclein (mRFP:: $\alpha$-SYN), green fluorescent tagged to $\alpha$-synuclein (GFP:: $\alpha$-SYN), GFP tagged to synapsin-I (GFP::SYS-I), GFP tagged to GAPDH (GFP::GAPDH), and amyloid precursor protein tagged to yellow fluorescent protein (APP::YFP), have been described previously (Kaether et al., 2000; Roy et al., 2007).

Cell culture, transfection, and pharmacologic experiments. Hippocampal cultures were obtained from brains of embryonic day 18 (E18)E19 C57BL/6 mice following standard protocols. Briefly, dissociated cells were plated at a density of 50,000 cells $/ \mathrm{cm}$ in poly-D-lysine-coated glassbottom culture dishes (Mattek, Ashland, MA) and maintained in Neurobasal/B27 plating media (Invitrogen) supplemented with $0.5 \mathrm{~mm}$ glutamine. Cells were transfected with the respective construct(s) using Lipofectamine-2000 (Invitrogen) at $7-8 \mathrm{~d}$ in vitro (DIV) according to the manufacturer's instructions, and transport studies were performed at 10-12 DIV. All animal studies were performed in accordance with University of Pennsylvania guidelines. For the drug experiments, appropriate doses of latrunculin, vinblastine, or nocodazole were added to the plating as well as the imaging media. For some experiments, latrunculin-treated neurons were imaged to visualize transport, immediately fixed/extracted thereafter with the cytoskeleton-stabilizing buffer, and then immediately stained with fluorescent phalloidin. This allowed us to visualize the actin cytoskeleton specifically in the axons wherein we observed transport. For some experiments, dissociated sympathetic neurons were isolated from newborn rat pups as described previously (Francis et al., 2005). Details of transfection and imaging in these neurons have also been published previously (Roy et al., 2000; Francis et al., 2005). 
Microscopy, live-cell imaging, and image analysis. Live-cell imaging, microscopy, and image analysis were performed as previously described (Roy et al., 2007), except that Hibernate E (low fluorescence; Brainbits, Springfield, IL) supplemented with 2\% B27, 2 mm Glutamax, 0.4\% D-glucose, and $37.5 \mathrm{~mm} \mathrm{NaCl}$ was used for imaging (Trivedi et al., 2007) instead of the L-15based media described previously. We improved our detection of transported particles significantly by focusing on thin axons with low background fluorescence of exogenously expressed proteins. All transport analysis was performed using kymographs as described, using the "multi-line" tool in MetaMorph (Molecular Devices, Sunnyvale, CA) software (Roy et al., 2007). Images and movies were scaled to maximize bit depth without any additional manipulation. Our dual-cam system for simultaneously visualizing red/green fluorophores was described previously (Roy et al., 2007). All statistical analyses were performed using Prism software (Graphpad, San Diego, CA). To allow comparison of frequencies between different experimental groups, we standardized our imaging methods by focusing on axons that traverse the length of the chip, thus capturing movies from approximately the same length of axon $(\approx 50-60 \mu \mathrm{m})$ in each experiment.

\section{Results}

Transport of $\alpha$-synuclein in SCb is

interrupted by pauses of

variable duration

Since our initial report on live-cell imaging of $\alpha$-synuclein transport (Roy et al., 2007), we have refined our methods, allowing us to obtain time-lapse movies with greater clarity, mainly by enhancing the signal-to-noise ratio of the moving cargoes. We achieved this by focusing on thin axons that had a very low diffuse fluorescence of the transfected proteins. In such axons, the vectorial movement of the punctate $\mathrm{SCb}$ cargoes is usually clear and easily quantified. We also found that the use of mRFP-tagged proteins gives a better signal-to-noise ratio than enhanced GFP-tagged proteins, probably because of their low autofluorescence. One concern with the use of mRFP-tagged proteins is the possibility of aggregation of the fluorescent protein despite its monomeric state, especially when tagged to membraneassociated proteins. In our system, however, we find that exogenously expressed mRFP:: $\alpha$-SYN is highly enriched at presynapses in mature cultures and also colocalizes precisely with the endogenous axonal mouse $\alpha$-synuclein as shown in supplemental Figure 1, $A$ and $B$ (available at www.jneurosci.org as supplemental material), indicating that the tagged $\alpha$-synuclein is behaving like its endogenous counterpart. Furthermore, we obtained similar results with a GFP:: $\alpha$-SYN vector, and in axons expressing both mRFP:: $\alpha$-SYN and GFP:: $\alpha-S Y N$, the tagged proteins precisely colocalize with each other and move together in the axons, both anterogradely and retrogradely. An example of one such kymograph is shown in

B.
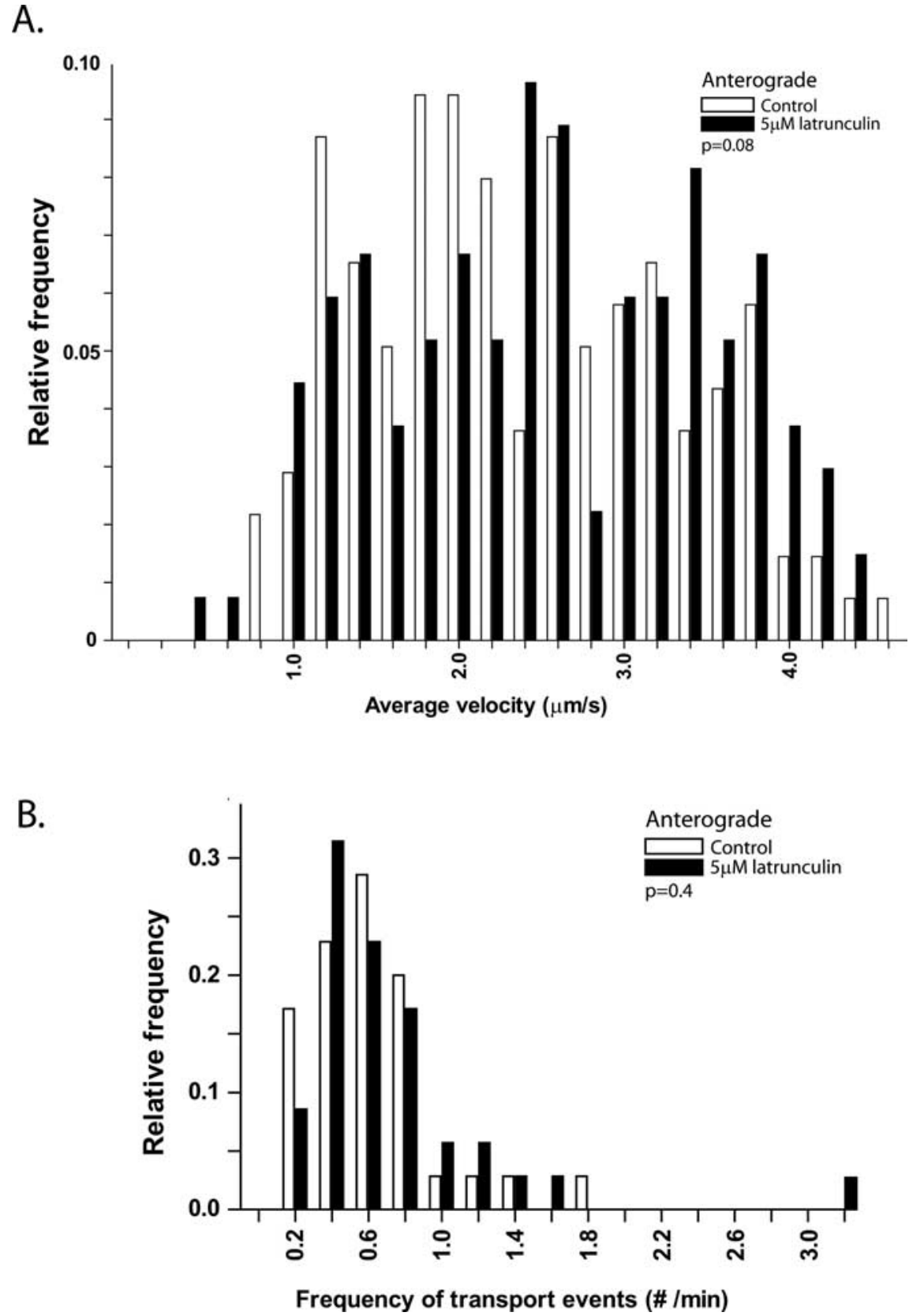

Figure 2. Quantitative parameters of $\alpha$-synuclein transport in F-actin-depleted axons. $\boldsymbol{A}$, Cultured hippocampal neurons were treated with $5 \mu \mathrm{m}$ latrunculin for $18-24 \mathrm{~h}$ to deplete actin filaments, and $\alpha$-synuclein transport was quantified in these axons. Frequency histograms of average anterograde velocities of moving mRFP.: $\alpha$-SYN particles (excluding pauses) in control (white bars) and latrunculin-treated (black bars) axons are shown. Note that in both groups, $\alpha$-synuclein moves rapidly at a broad range of velocities, and the two groups are statistically similar ( $p=0.8$, Mann-Whitney test, two tailed). $\boldsymbol{B}$, Particles in both control and latrunculin-treated groups move with a range of transport frequencies, and the two groups are statistically similar ( $p=0.4$, Mann-Whitney test, two tailed).

supplemental Figure 1C (available at www.jneurosci.org as supplemental material).

Figure $1 A$ shows several examples of kymographs obtained from imaging mRFP:: $\alpha$-SYN in axons. In our kymographs, anterograde vectorial transport is represented by diagonal lines moving from bottom left to top right, and pauses are represented by horizontal lines (see Materials and Methods for details). Note that these objects undergo episodes of rapid transport (green arrowheads) interrupted by quiescent periods of little or no movement (red asterisks), as described previously for slowcomponent cargoes (Roy et al., 2000, 2007; Wang et al., 2000; 
Table 1. Quantitative data comparing mRFP:: $\alpha$-SYN transport in control and latrunculin-treated axons in cultured hippocampal neurons

\begin{tabular}{lllll}
\hline & Total imaging time $(\mathrm{min})$ & No. of moving particles & $\begin{array}{l}\text { Frequency } \\
\text { (no./min) }\end{array}$ & Average velocity $(\mu \mathrm{m} / \mathrm{s})$ \\
\hline Control & 226 & 137 & $0.62 \pm 0.37$ & $2.37 \pm 0.9$ \\
Latrunculin $(5 \mu \mathrm{M})$ & 212 & 139 & $0.74 \pm 0.53$ & $2.31 \pm 0.9$ \\
\hline
\end{tabular}

All data are derived from kymograph analysis. Average velocities (when moving) are expressed as mean \pm SD. No., Number.

Wang and Brown, 2002). We also noted that the pauses were quite variable in duration. Although many particles either did not pause or paused only briefly $(<30 \mathrm{~s})$, other particles paused for longer times, often for several minutes (Fig. $1 A$, multiple red asterisks). We also saw particles that were immobile for most of the duration of the sequence but later showed bursts of vectorial movement indistinguishable from other moving particles (Fig. $1 A$, bottom). Particles showing both transient and prolonged pauses were also observed (Fig. $1 \mathrm{~A}$, double arrowheads). We also commonly noted objects that did not move throughout the duration of the sequences (typically 5-8 min). Given the natural variation in the duration of the pauses together with the fact that mRFP:: $\alpha$-SYN objects commonly pause for several minutes between movement events, it seems reasonable that some and perhaps all of the observed objects that did not move are simply paused in their translocation within the axon. In our previous work, we also showed that the frequency of moving SCb cargoes was relatively low ( 1 object every $\approx 2 \mathrm{~min}$ ) and notably much lower than the frequency of moving FC cargoes. We now report similar findings with APP, a classic marker of the FC (Koo et al., 1990). Figure $1 B$ shows representative kymographs from $\alpha$-synuclein and APP transport. Note the marked difference in the frequencies between the two.

\section{SCb transport is dependent, not on actin filaments, but on microtubules}

To test whether actin filaments are required for $\mathrm{SCb}$ transport, we evaluated mRFP:: $\alpha$-SYN transport in neurons depleted of actin filaments by treatment with latrunculin. To maximally deplete actin filaments, neurons were treated with $5 \mu \mathrm{M}$ latrunculin for $18-24$ h. Previous work has shown that these conditions are sufficient to depolymerize $\geq 90 \%$ of F-actin in cultured axons, and we obtained comparable results (supplemental Fig. 2, available at www.jneurosci.org as supplemental material) (Allison et al., 1998; Francis et al., 2005). Transport continued unabated in these actin-depleted axons, with velocities and frequencies indistinguishable from those in control neurons (Fig. 2A, B, Table 1). To confirm that our latrunculin treatments effectively depleted actin filaments specifically in the imaged axons, several axons were fixed after imaging and then stained with fluorescein-phalloidin immediately thereafter to reveal remaining actin filaments, if any. In all cases, these retrospective analyses confirmed depletion of actin filaments in the specific axons that showed vectorial movements. A representative example of these data is shown in Figure $3 A$.

Given that vectorial $\alpha$-synuclein transport does not depend on F-actin, it seems likely that these movements instead require intact microtubules. To test the role of microtubules in $\mathrm{SCb}$ transport, we disrupted microtubules in our system using $5 \mu \mathrm{M}$ vinblastine for $3 \mathrm{~h}$ and visualized $\alpha$-synuclein transport in these drug-treated axons. We have previously shown that these conditions are sufficient to disrupt microtubules in cultured sympathetic neurons without any detectable change in cell viability (Francis et al., 2005), and we obtained similar results with our hippocampal cultures (see supplemental Fig. 2, available at www.jneurosci.org as supplemental material). Microtubule disruption caused approximately sevenfold inhibition of $\alpha$-synuclein transport, with only four vectorial movements seen in $2780 \mathrm{~s}$ of imaging ( 1 movement every $695 \mathrm{~s}$ of imaging time). Short-range mergingsplitting of mRFP:: $\alpha$-SYN-labeled vesiclelike structures continued in these microtubule-disrupted axons, suggesting that other physiologic mechanisms were preserved. A representative example of these data is shown in Figure 4. These results establish that $\alpha$-synuclein transport in SCb is microtubule dependent. A recent study also showed that $\alpha$-synuclein binds to lipid rafts at presynapses (Fortin et al., 2004), so we asked whether $\alpha$-synuclein transport in SCb was mediated via its association with these rafts. To do this, we used $10 \mu \mathrm{g} / \mathrm{ml}$ nystatin to acutely disrupt rafts in our cultures as described previously (Fortin et al., 2004) and then visualized mRFP:: $\alpha$-SYN transport. We observed $\alpha$-synuclein transport before and after adding nystatin, indicating that disruption of lipid rafts does not completely prevent synuclein transport (data not shown).

Finally, we also examined the transport of $\mathrm{SCb}$ proteins in a different system to evaluate the generality of the observations with cultured hippocampal neurons by using cultured sympathetic neurons obtained from newborn rats. Because $\alpha$-synuclein is not expressed reliably in these cultures (our unpublished data), we focused on synapsin-I, using a GFP-tagged synapsin-I (GFP:: SYS-I) to label SCb cargoes for live-cell imaging. In every respect examined, GFP::SYS-I transport in these neurons closely resembled that of hippocampal neurons. Specifically, synapsin-I is organized into discrete, punctate structures that are transported rapidly at rates of $1-2 \mu \mathrm{m} / \mathrm{s}$, but transport is infrequent and interrupted by pauses. Disruption of actin filaments with latrunculin had no statistically significant effect on the rate or frequency of synapsin-I transport, thus confirming in a separate system that transport of this $\mathrm{SCb}$ protein does not require actin filaments (Table 2). However, no synapsin-I movements were seen on disrupting microtubules with vinblastine or nocodazole $(0$ movements in $87 \mathrm{~min}$ of imaging time).

\section{Actin filaments do not act as a carrier for multiple $\mathrm{SCb}$ proteins}

To rigorously assess whether actin filaments function as a carrier or scaffold for $\mathrm{SCb}$ proteins, we next determined whether actin filament depletion disrupts the cotransport of SCb proteins. Using our dual-cam system, we have previously shown that $\alpha$-synuclein, synapsin-I, and GAPDH are cotransported as components of an SCb cargo complex (Roy et al., 2007), and classical transport studies using radioisotopic labeling suggest that many more SCb proteins are cotransported as components of discrete SCb cargoes as well (Garner and Lasek, 1982). As mentioned previously, it has been suggested that actin filaments are at the core of these cargo complexes, serving as a scaffold to bind directly or indirectly the other SCb proteins. If this is correct, then disruption of actin filaments should disrupt the complex and prevent the cotransport of the component proteins. We tested this by evaluating the cotransport of $\alpha$-synuclein, synapsin-I, and GAPDH in hippocampal neurons depleted of actin filaments by treatment with latrunculin. As shown in Figure $5 A$, cotransport of red/green-labeled $\alpha$-synuclein/synapsin-I continued despite actin filament depletion. Recently, it was reported that a $10 \times$ higher dose of latrunculin $(50 \mu \mathrm{M})$ for much shorter times $(2-10$ 


\section{A. mRFP:: $\alpha$-syn transport in f-actin depleted axons}
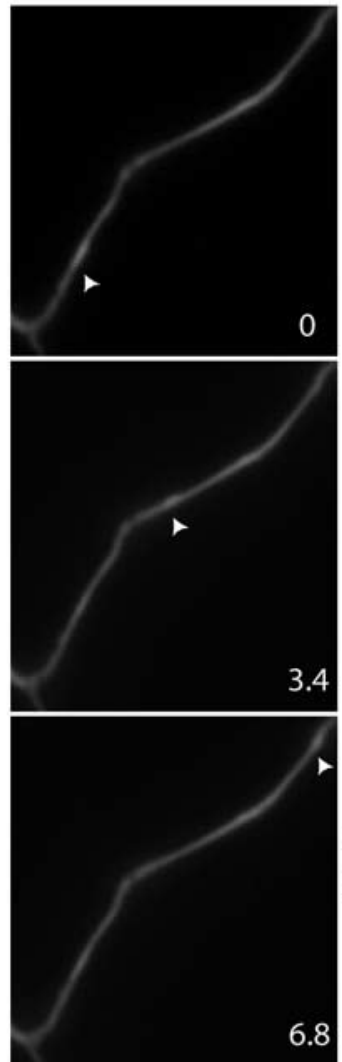

.

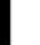

Kymograph of moving particle on left

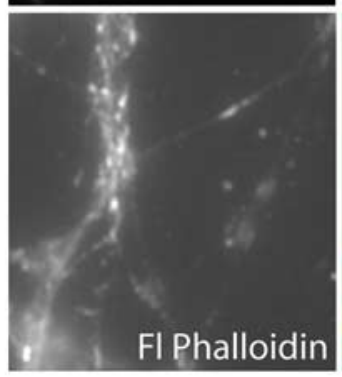

68
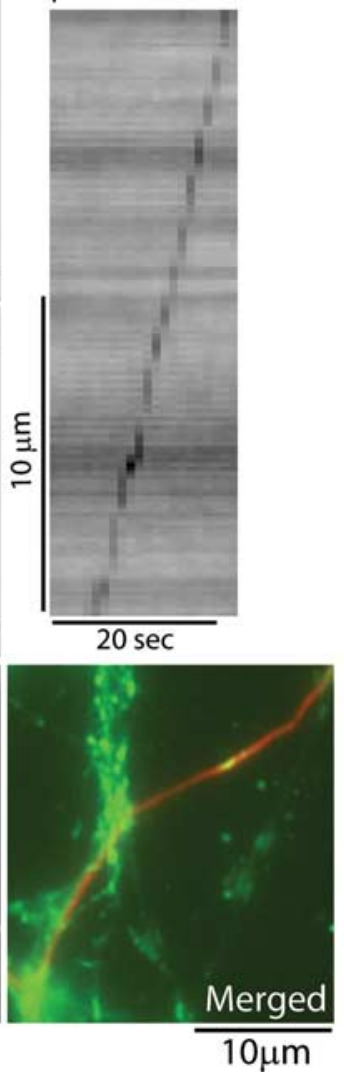

\section{B. Kymographs of mRFP:: $\alpha$-syn transport in f-actin depleted axons}

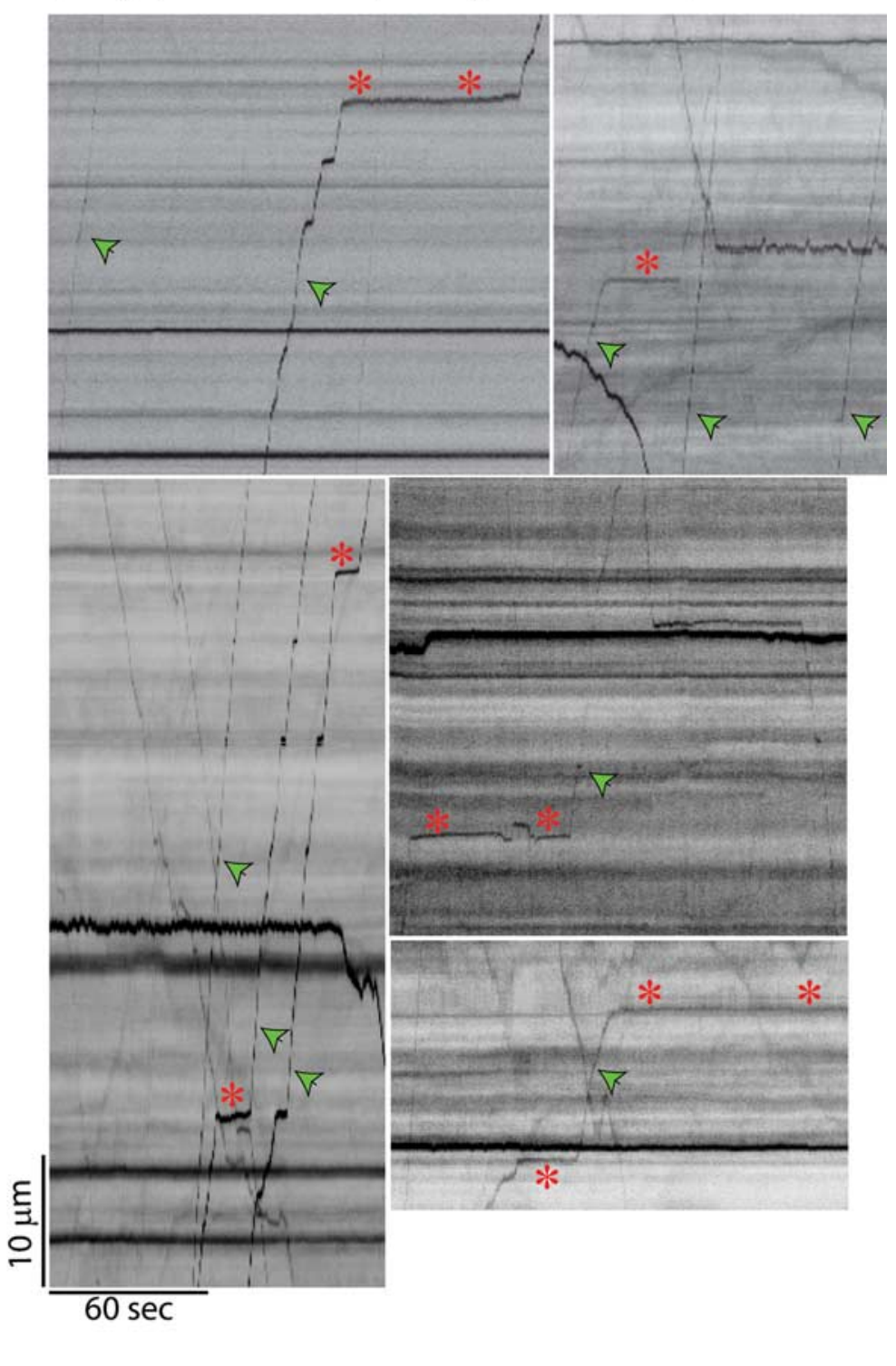

Figure 3. Dynamics of $\alpha$-synuclein transport in F-actin-depleted axons. $A$, Cultured hippocampal neurons were transfected with mRFP:: $\alpha$-SYN and treated with latrunculin for $18-24 \mathrm{~h}$. One such transfected axon was imaged to visualize $\alpha$-synuclein transport, fixed/extracted immediately afterward in a cytoskeleton-stabilizing buffer (see Materials and Methods), and then stained with fluorescein-phalloidin (FI Phalloidin) to reveal F-actin in the same axon. Selected frames from the time lapse are shown at left, and the phalloidin stain is shown at the bottom (merged image on right). Note vectorial transport of mRFP:: $\alpha$-SYN particle (arrowhead) in the actin-depleted axon, as depicted by the kymograph to the right. $\boldsymbol{B}$, Representative $\alpha$-synuclein kymographs obtained from imaging latrunculin-treated axons. Note that the movement is rapid (green arrowheads) with pauses (red asterisks), reminiscent of the movements seen in control axons. Scale bars for the kymographs are shown at the bottom left of the kymograph in $\boldsymbol{A}$ and the group of kymographs in $\boldsymbol{B}$.

min) can cause an acute disruption of F-actin in cultured hippocampal neurons (Sankaranarayanan et al., 2003). To provide a more thorough assessment of possible effects of actin filament disruption on SCb transport, we tested this acute treatment regimen as well, and even under these conditions, cotransport of $\alpha$-synuclein, synapsin-I, and GAPDH continues (Fig. 4B). Thus, we conclude that actin filaments are not required as a carrier for the cotransport of these SCb proteins.

\section{Discussion}

Our purpose in the present studies was to evaluate the role of actin filaments in SCb transport. Actin filaments could participate in SCb transport in two different ways: (1) as a component of the motor system that powers SCb transport and (2) as a scaffold for binding and organizing the transport of $\mathrm{SCb}$ proteins. The possibility that actin filaments could have one or both of these functions evolved initially from the identification of actin and myosin in SCb, and this notion was reinforced further by subsequent observations demonstrating that several SCb proteins had actin-binding activity. However, the concept lacked definitive experimental support, and for this reason, we addressed this issue here. Briefly, we focused on three SCb proteins, $\alpha$-synuclein, synapsin-I, and GAPDH, because we previously showed that they are components of a protein complex transported in $\mathrm{SCb}$ (Roy et al., 2007). The present results show unequivocally that the transport of these proteins in SCb continues after pharmacologic treatments that deplete actin filaments and that their transport in these actin-depleted axons is indistinguishable from that in control neurons. Notably, these three proteins continue to undergo 


\section{mRFP:: $\alpha$-syn in vinblastine treated axons}

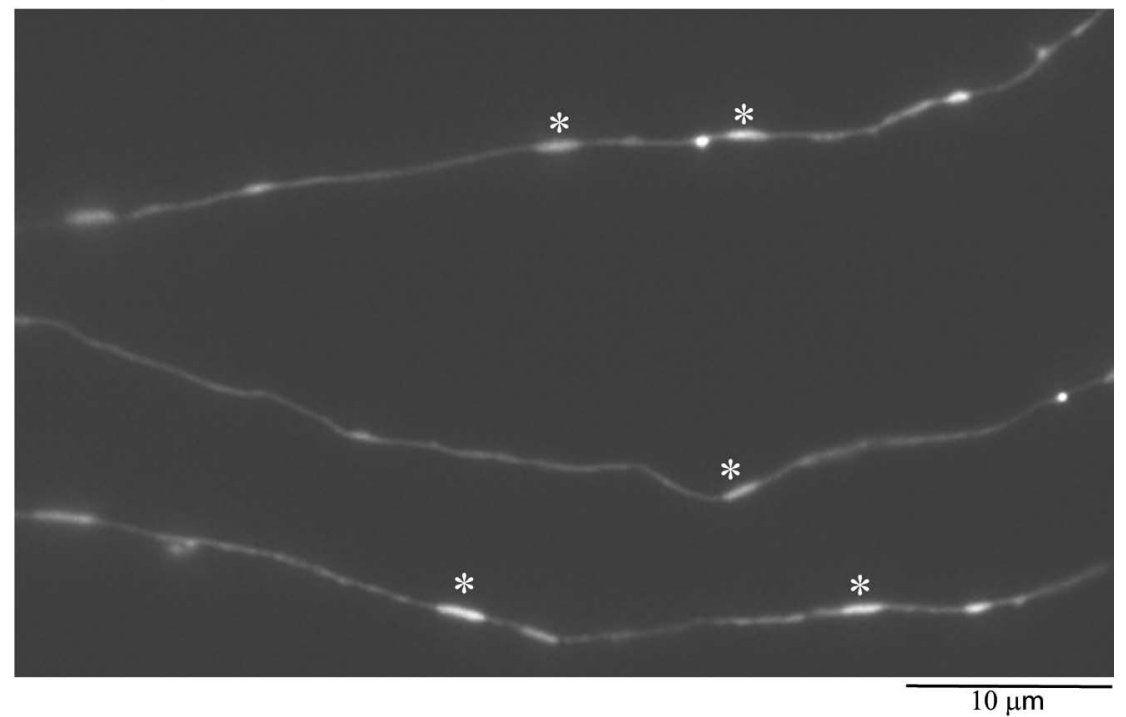

Kymograph, upper axon

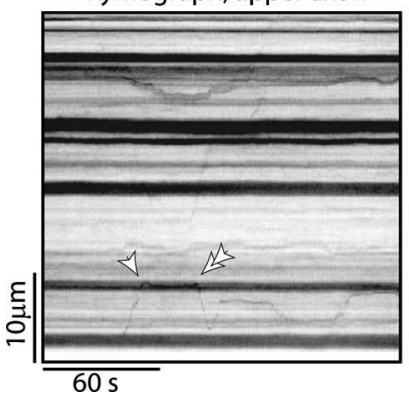

Kymograph, middle axon

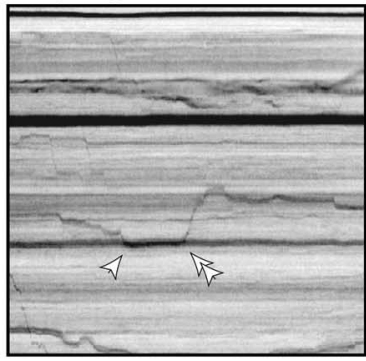

Kymograph, lower axon

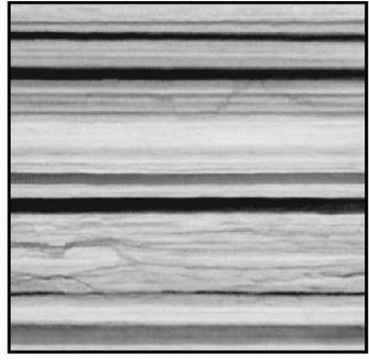

Figure 4. SCb transport is dependent on microtubules. Cultured hippocampal neurons were transfected with mRFP::: $\alpha$-SYN and then treated with vinblastine for $3 \mathrm{~h}$ to disrupt microtubules. Vectorial movements were greatly diminished under these conditions. A representative image from one such time lapse is shown. Note that elongated clusters of mRFP:: $\alpha$-SYN were seen along isolated axons that probably represent stalled $\alpha$-synuclein cargoes (asterisks). The kymographs (bottom) from the respective axons above demonstrate that particles do not show any appreciable vectorial transport. Note that merging/splitting of vesicle-like $\alpha$-synuclein clusters (single and double arrowheads, respectively) continues in these axons, leading to short-range movements.

Table 2. Quantitative data comparing GFP::SYS-I transport in control and latrunculin-treated axons in cultured sympathetic neurons

\begin{tabular}{lllll}
\hline & $\begin{array}{l}\text { Total imaging time } \\
\text { (min) }\end{array}$ & $\begin{array}{l}\text { No. of moving } \\
\text { particles }\end{array}$ & $\begin{array}{l}\text { Frequency } \\
\text { (no./min) }\end{array}$ & $\begin{array}{l}\text { Average velocity } \\
(\mu \mathrm{m} / \mathrm{s})\end{array}$ \\
\hline Control & 136 & 74 & $0.44 \pm 0.25$ & $1.7 \pm 0.7$ \\
Latrunculin $(5 \mu \mathrm{M})$ & 72 & 30 & $0.47 \pm 0.35$ & $2.0 \pm 0.6$ \\
\hline
\end{tabular}

All data are derived from kymograph analysis. Average velocities (when moving) are expressed as mean \pm SD. No., Number.

cotransport in these actin-depleted axons. Thus, although we cannot completely exclude the possibility that SCb transport is different in adult neurons that are more enriched in F-actin, we provide compelling experimental data to support our conclusion that actin filaments are neither essential components of the motor mechanisms that transport these proteins nor essential as a scaffold for their cotransport in our model system.

To what extent do the present findings extend to the transport of the other SCb proteins? This question arises because $\mathrm{SCb}$ transports $>200$ different proteins, whereas our studies have examined only three of these. Although the available evidence does not allow us to rigorously answer this question, several observations suggest that the present findings extend well beyond these three proteins. Although direct evidence for the cotransport of SCb exists only for the three proteins studied here (Fig. 5) (see also Roy et al., 2007), several indirect observations indicate that the proteins of $\mathrm{SCb}$ are organized into much larger multicomponent complexes that are transported in axons. First, radioisotopic labeling studies have provided data suggesting that many of the $\mathrm{SCb}$ proteins move together as if they comprised discrete structures that are transported in axons (Willard et al., 1974, 1979; Black and Lasek, 1980; Tytell et al., 1981; Garner and Lasek, 1981, 1982). This possibility was reinforced by studies in which large subsets of SCb proteins were isolated by subcellular fractionation (Lorenz and Willard, 1978) or nondenaturing immunoprecipitation (Black et al., 1991). Finally, a review of the literature reveals that many other $\mathrm{SCb}$ proteins interact with the three proteins that are cotransported in our system. For example, a recent study investigating $\alpha$-synuclein binding partners identified several known SCb proteins, including dynein, synapsin-I, components of the ubiquitin system, annexin, and heat shock cognate-70 (hsc70) (Woods et al., 2007). Several other chaperones and metabolic enzymes were also isolated as $\alpha$-synuclein binding partners in this assay, and although these are not among the limited list of known SCb proteins, we note that such proteins typically move in SCb. Furthermore, interactions of synapsin with the $\mathrm{SCb}$ proteins spectrin and calmodulin have been reported (Baines and Bennett, 1985; Nicol et al., 1997), and the SCb protein MAP-1B was shown to bind to $\mathrm{GAPDH}$, spectrin, and clathrin, all $\mathrm{SCb}$ proteins (Cueille et al., 2007). It is also known that hsc70 exists as a complex with clathrin and a variety of other SCb proteins (Black et al., 1991). Given our data showing that $\alpha$-synuclein, synapsin-I, and GAPDH are cotransported as a complex and the above described interactions between the three proteins that we studied and other known SCb members, we infer that all these proteins are a part of a transported SCb complex that depends on microtubules rather than on the actin network.

Clearly, more work is required to understand the extent and nature of interactions among the proteins in SCb. In particular, the available information does not conclusively reveal the number of discrete complexes transported by $\mathrm{SCb}$ or the specific composition of these complexes. It is also unknown whether these complexes are simply carriers for the delivery of sets of SCb proteins to the axon and axon terminal or whether $\mathrm{SCb}$ proteins are organized into discrete complexes on the basis of their functional specializations, as reported for FC cargoes (Sytnyk et al., 2004). Nonetheless, the considerations summarized above indicate that the potential interactions among SCb proteins are extensive and are consistent with the possibility that $\alpha$-synuclein, synapsin-I, and GAPDH are only three of a much larger number of proteins that comprise a discrete SCb cargo. Also, the studies above showing interactions between various $\mathrm{SCb}$ members coupled with our 


\section{A. Prolonged latrunculin treatment: $5 \mu \mathrm{M}$ for $18-24 \mathrm{~h}$}

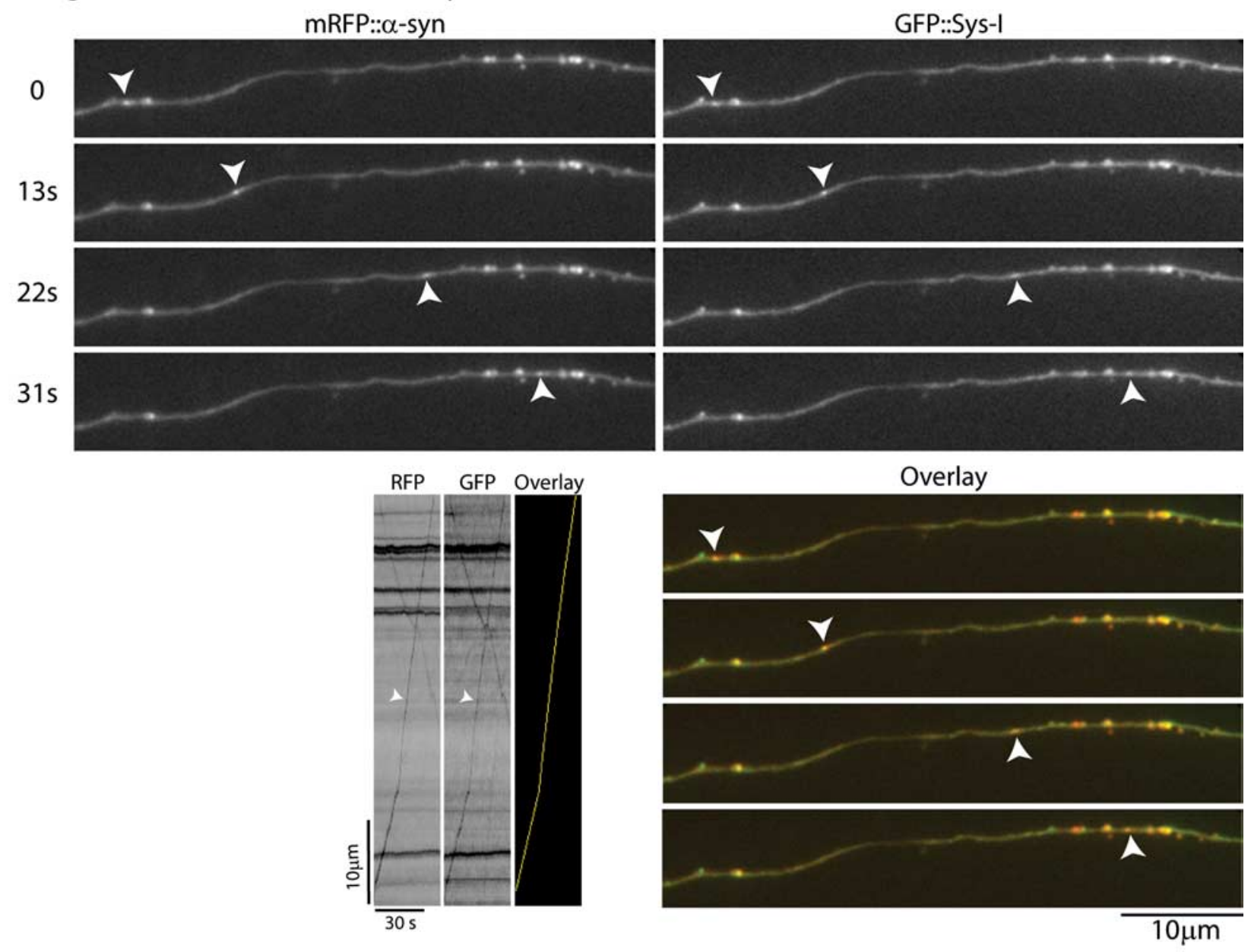

\section{B Acute latrunculin treatment: $50 \mu \mathrm{M}$ latrunculin for $<10 \mathrm{~min}$}
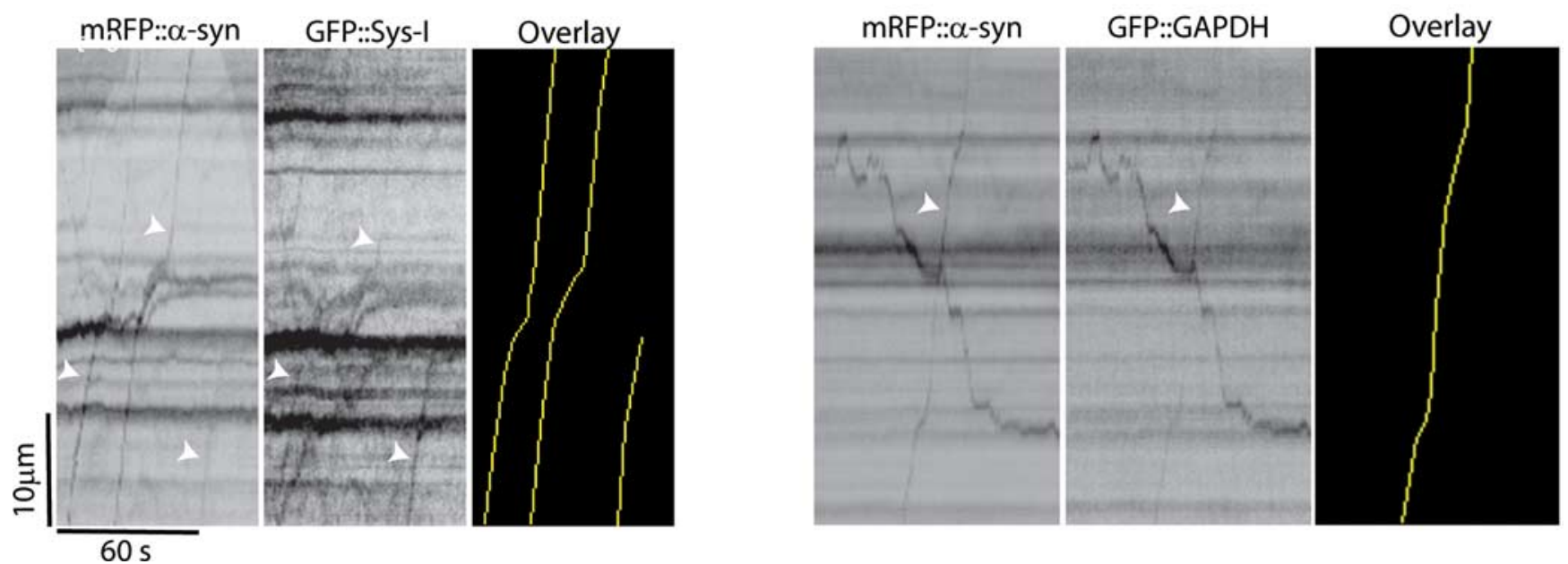

Figure 5. Cotransport of SCb proteins continue in F-actin-depleted axons. $\boldsymbol{A}$, Cultured hippocampal neurons were double transfected with mRFP:: $\alpha$-SYN and GFP::SYS-I, respectively, treated with $5 \mu \mathrm{m}$ latrunculin for $18-24 \mathrm{~h}$, and visualized by simultaneous dual-color video microscopy. Selected frames from a time-lapse sequence show a single mRFP:: $\alpha$-SYN particle and a GFP::SYS-I particle cotransported anterogradely (arrowhead, yellow in merged). Kymographs generated from the movies in the red/green channel are shown as well. Note that the two fusion proteins colocalize precisely during transport, suggesting that the $\alpha$-synuclein/synapsin-I complex is not disrupted by actin depletion. B, Axons double transfected with mRFP:: $\alpha$-SYN and GFP-tagged synapsin-I or GAPDH were treated with a high dose $(50 \mu \mathrm{m})$ of latrunculin, known to cause acute disruption of F-actin, and then imaged with dual-color simultaneous video microscopy. Note that cotransport of $\alpha$-synuclein and synapsin-I or $\alpha$-synuclein and GAPDH continues under these conditions as well. 
observations that actin is not required for maintaining the $\mathrm{SCb}$ complex suggest that innate affinities among SCb members (and not actin) may be sufficient in maintaining the integrity of these complexes. Additional focused studies are needed to address these issues.

Whereas actin disruption has no detectable effect on $\mathrm{SCb}$ transport in two different systems, disruption of microtubules with either nocodazole or vinblastine brings SCb transport to a halt. These findings focus attention on microtubules and microtubule motors in powering the movement of SCb cargoes. Presumably one or more of the kinesin family of motors mediates anterograde transport of $\mathrm{SCb}$, whereas dynein mediates retrograde transport. The identity of the specific motors involved is not certain, although biochemical evidence exists for the interaction of kinesin-1 (conventional kinesin) with $\alpha$-synuclein (Utton et al., 2005; Woods et al., 2007), raising the possibility that this motor contributes to anterograde $\mathrm{SCb}$ transport. In this regard, detailed analyses of kinesin-1 transport in pulse-chase studies failed to detect any kinesin-1 moving with SCb (Elluru et al., 1995). If kinesin-1 does contribute to SCb transport, then one possibility is that its association with the $\mathrm{SCb}$ cargoes is transient, thus accounting for the absence of detectable amounts of this motor in SCb. Perhaps the transient association of kinesin- 1 with $\mathrm{SCb}$ cargoes contributes to the observation that these cargoes pause often for relatively prolonged times during their transit in the axon. Kinesin-1 may dissociate from the cargo so that the cargo remains paused until engaged again by another motor. In this regard, regulation of vesicle transport by kinesin- 1 involves site-specific phosphorylation events that control the attachment and detachment of kinesin to its cargoes, and thereby whether the cargo moves or stops (Lee and Hollenbeck, 1995; Morfini et al., 2002, 2004; Horiuchi et al., 2005). Key issues for the future include identifying the specific motors that power $\mathrm{SCb}$ transport and determining how they are regulated to produce the stopand-go movement typical of SCb cargoes.

\section{References}

Allison DW, Gelfand VI, Spector I, Craig AM (1998) Role of actin in anchoring postsynaptic receptors in cultured hippocampal neurons: differential attachment of NMDA versus AMPA receptors. J Neurosci 18:2423-2436.

Bahler M, Greengard P (1987) Synapsin I bundles F-actin in a phosphorylation-dependent manner. Nature 326:704-707.

Baines AJ, Bennett V (1985) Synapsin I is a spectrin-binding protein immunologically related to erythrocyte protein 4.1. Nature 315:410-413.

Baitinger C, Willard M (1987) Axonal transport of synapsin I-like proteins in rabbit retinal ganglion cells. J Neurosci 7:3723-3735.

Black MM, Lasek RJ (1979) Axonal transport of actin: slow component b is the principal source of actin for the axon. Brain Res 171:401-413.

Black MM, Lasek RJ (1980) Slow components of axonal transport: two cytoskeletal networks. J Cell Biol 86:616-623.

Black MM, Chestnut MH, Pleasure IT, Keen JH (1991) Stable clathrin: uncoating protein (hsc70) complexes in intact neurons and their axonal transport. J Neurosci 11:1163-1172.

Bourke GJ, El Alami W, Wilson SJ, Yuan A, Roobol A, Carden MJ (2002) Slow axonal transport of the cytosolic chaperonin CCT with Hsc73 and actin in motor neurons. J Neurosci Res 68:29-35.

Brady ST, Tytell M, Heriot K, Lasek RJ (1981) Axonal transport of calmodulin: a physiologic approach to identification of long-term associations between proteins. J Cell Biol 89:607-614.

Bray JJ, Fernyhough P, Bamburg JR, Bray D (1992) Actin depolymerizing factor is a component of slow axonal transport. J Neurochem 58:2081-2087.

Cueille N, Blanc CT, Riederer IM, Riederer BM (2007) Microtubuleassociated protein $1 \mathrm{~B}$ binds glyceraldehyde-3-phosphate dehydrogenase. J Proteome Res 6:2640-2647.

Dillman III JF, Dabney LP, Karki S, Paschal BM, Holzbaur EL, Pfister KK
(1996) Functional analysis of dynactin and cytoplasmic dynein in slow axonal transport. J Neurosci 16:6742-6752.

Elluru RG, Bloom GS, Brady ST (1995) Fast axonal transport of kinesin in the rat visual system: functionality of kinesin heavy chain isoforms. Mol Biol Cell 6:21-40.

Fortin DL, Troyer MD, Nakamura K, Kubo S, Anthony MD, Edwards RH (2004) Lipid rafts mediate the synaptic localization of $\alpha$-synuclein. J Neurosci 24:6715-6723.

Francis F, Roy S, Brady ST, Black MM (2005) Transport of neurofilaments in growing axons requires microtubules but not actin filaments. J Neurosci Res 79:442-450.

Garner JA, Lasek RJ (1981) Clathrin is axonally transported as part of slow component b: the microfilament complex. J Cell Biol 88:172-178.

Garner JA, Lasek RJ (1982) Cohesive axonal transport of the slow component b complex of polypeptides. J Neurosci 2:1824-1835.

Giasson BI, Jakes R, Goedert M, Duda JE, Leight S, Trojanowski JQ, Lee VMY (2000) A panel of epitope-specific antibodies detects protein domains distributed throughout human $\alpha$-synuclein in Lewy bodies of Parkinson's disease. J Neurosci Res 59:528-533.

Heriot K, Gambetti P, Lasek RJ (1985) Proteins transported in slow components $\mathrm{a}$ and $\mathrm{b}$ of axonal transport are distributed differently in the transverse plane of the axon. J Cell Biol 100:1167-1172.

Horiuchi D, Barkus RV, Pilling AD, Gassman A, Saxton WM (2005) APLIP1, a kinesin binding JIP-1/JNK scaffold protein, influences the axonal transport of both vesicles and mitochondria in Drosophila. Curr Biol 15:2137-2141.

Kaether C, Skehel P, Dotti CG (2000) Axonal membrane proteins are transported in distinct carriers: a two-color video microscopy study in cultured hippocampal neurons. Mol Biol Cell 11:1213-1224.

Koo EH, Sisodia SS, Archer DR, Martin LJ, Weidemann A, Beyreuther K, Fischer P, Masters CL, Price DL (1990) Precursor of amyloid protein in Alzheimer disease undergoes fast anterograde axonal transport. Proc Natl Acad Sci USA 87:1561-1565.

Lasek RJ, Garner JA, Brady ST (1984) Axonal transport of the cytoplasmic matrix. J Cell Biol 99:212s-221s.

Lee KD, Hollenbeck PJ (1995) Phosphorylation of kinesin in vivo correlates with organelle association and neurite outgrowth. J Biol Chem 270:5600-5605.

Levine J, Willard M (1981) Fodrin: axonally transported polypeptides associated with the internal periphery of many cells. J Cell Biol 90:631-642.

Li W, Hoffman PN, Stirling W, Price DL, Lee MK (2004) Axonal transport of human alpha-synuclein slows with aging but is not affected by familial Parkinson's disease-linked mutations. J Neurochem 88:401-410.

Lorenz T, Willard M (1978) Subcellular fractionation of intra-axonally transport polypeptides in the rabbit visual system. Proc Natl Acad Sci USA 75:505-509.

Ma D, Himes BT, Shea TB, Fischer I (2000) Axonal transport of microtubule-associated protein 1B (MAP1B) in the sciatic nerve of adult rat: distinct transport rates of different isoforms. J Neurosci 20:2112-2120.

Mercken M, Fischer I, Kosik KS, Nixon RA (1995) Three distinct axonal transport rates for tau, tubulin, and other microtubule-associated proteins: evidence for dynamic interactions of tau with microtubules in vivo. J Neurosci 15:8259-8267.

Minaschek G, Groschel-Stewart U, Blum S, Bereiter-Hahn J (1992) Microcompartmentation of glycolytic enzymes in cultured cells. Eur J Cell Biol $58: 418-428$.

Morfini G, Szebenyi G, Elluru R, Ratner N, Brady ST (2002) Glycogen synthase kinase 3 phosphorylates kinesin light chains and negatively regulates kinesin-based motility. EMBO J 21:281-293.

Morfini G, Szebenyi G, Brown H, Pant HC, Pigino G, DeBoer S, Beffert U, Brady ST (2004) A novel CDK5-dependent pathway for regulating GSK3 activity and kinesin-driven motility in neurons. EMBO J 23:2235-2245.

Moss ML, Palmer RE, Kuzmic P, Dunlap BE, Henzel W, Kofron JL, Mellon WS, Royer CA, Rich DH (1992) Identification of actin and HSP 70 as cyclosporin $\mathrm{A}$ binding proteins by photoaffinity labeling and fluorescence displacement assays. J Biol Chem 267:22054-22059.

Nicol S, Rahman D, Baines AJ (1997) Ca2+-dependent interaction with calmodulin is conserved in the synapsin family: identification of a highaffinity site. Biochemistry 36:11487-11495.

Nixon RA, Fischer I, Lewis SE (1990) Synthesis, axonal transport, and turn- 
over of the high molecular weight microtubule-associated protein MAP $1 \mathrm{~A}$ in mouse retinal ganglion cells: tubulin and MAP 1A display distinct transport kinetics. J Cell Biol 110:437-448.

Roy S, Coffee P, Smith G, Liem RK, Brady ST, Black MM (2000) Neurofilaments are transported rapidly but intermittently in axons: implications for slow axonal transport. J Neurosci 20:6849-6861.

Roy S, Zhang B, Lee VM-Y, Trojanowski JQ (2005) Axonal transport defects: a common theme in neurodegenerative diseases. Acta Neuropathol (Berl) 109:5-13.

Roy S, Winton MJ, Black MM, Trojanowski JQ, Lee VM-Y (2007) Rapid and intermittent cotransport of slow component-b proteins. J Neurosci 27:3131-3138.

Sankaranarayanan S, Atluri PP, Ryan TA (2003) Actin has a molecular scaffolding, not propulsive, role in presynaptic function. Nat Neurosci 6:127-135.

Slaughter T, Wang J, Black MM (1997) Microtubule transport from the cell body into the axons of growing neurons. J Neurosci 17:5807-5819.

Sytnyk V, Leshchyns'ka I, Dityatev A, Schachner M (2004) Trans-Golgi network delivery of synaptic proteins in synaptogenesis. J Cell Sci 117:381-388.

Trivedi N, Jung P, Brown A (2007) Neurofilaments switch between distinct mobile and stationary states during their transport along axons. J Neurosci 27:507-516.

Tytell M, Black MM, Garner JA, Lasek RJ (1981) Axonal transport: each major rate component reflects the movement of distinct macromolecular complexes. Science 214:179-181.

Utton MA, Noble WJ, Hill JE, Anderton BH, Hanger DP (2005) Molecular motors implicated in the axonal transport of tau and alpha-synuclein. J Cell Sci 118:4645-4654.

Wang L, Brown A (2002) Rapid movement of microtubules in axons. Curr Biol 12:1496-1501.

Wang L, Ho CL, Sun D, Liem RK, Brown A (2000) Rapid movement of axonal neurofilaments interrupted by prolonged pauses. Nat Cell Biol 2:137-141.

Willard M (1977) The identification of two intra-axonally transported polypeptides resembling myosin in some respects in the rabbit visual system. J Cell Biol 75:1-11.

Willard M, Cowan WM, Vagelos PR (1974) The polypeptide composition of intra-axonally transported proteins: evidence for four transport velocities. Proc Natl Acad Sci USA 71:2183-2187.

Willard M, Wiseman M, Levine J, Skene P (1979) Axonal transport of actin in rabbit retinal ganglion cells. J Cell Biol 81:581-591.

Woods WS, Boettcher JM, Zhou DH, Kloepper KD, Hartman KL, Ladror DT, Qi Z, Rienstra CM, George JM (2007) Conformation-specific binding of $\alpha$-synuclein to novel protein partners detected by phage display and NMR spectroscopy. J Biol Chem 282:34555-34567.

Yuan A, Mills RG, Bamburg JR, Bray JJ (1999) Cotransport of glyceraldehyde-3-phosphate dehydrogenase and actin in axons of chicken motoneurons. Cell Mol Neurobiol 19:733-744. 